\title{
The effect of oxygen doping on the magnetism of Tb and Pr clusters
}

\author{
C. N. van Dijk, ${ }^{1, a)}$ Th. Rasing, ${ }^{1}$ A. Kirilyuk, ${ }^{1}$ J. Bowlan, ${ }^{2}$ A. Liang, ${ }^{2}$ and W. A. de Heer ${ }^{2}$ \\ ${ }^{1}$ Institute for Molecules and Materials, Radboud University Nijmegen, Nijmegen 6525 AJ, \\ The Netherlands \\ ${ }^{2}$ School of Physics, Georgia Institute of Technology, Atlanta, Georgia 30332, USA
}

(Presented 21 January 2010; received 11 November 2009; accepted 18 November 2009; published online 14 May 2010)

The magnetic moments and electric dipoles of $\mathrm{Tb}$ and Pr clusters are investigated using the SternGerlach deflection technique. The addition of a single oxygen atom induces an increase in the electric dipole of $\mathrm{Tb}_{\mathrm{N}}$ clusters, however the magnetic moment is largely not affected. In Pr neither the magnetic moment nor the electric dipole is affected. This raises questions as to the role of conduction electrons in the exchange interaction of rare earth clusters, and puts into doubt the validity of the Ruderman-Kittel-Kasuya-Yosida (RKKY) exchange mechanism in small systems.

() 2010 American Institute of Physics. [doi:10.1063/1.3358611]

\section{INTRODUCTION}

The field of magnetism in small systems is of both fundamental and applied interest. Of particular importance is gaining a complete understanding of the interaction of individual spins within a confined system. Clusters represent a unique opportunity to study the effects of magnetism on a small scale in a methodical manner using a bottom-up approach, where the number of atoms in the system can be systematically and monotonically increased as the bulk properties are approached.

Here rare earth metal clusters are studied free in vacuum, where no interactions with the environment or a surface can take place. We then add an oxygen dopant atom to the system in order to investigate the effects of contaminants on the magnetic order of small magnetic systems. This has important implications with regards to their resistance to contamination and long-term stability, and thus to potential applications in fields such as nanospintronics and patterned magnetic storage.

In this paper we study the effects of adding an oxygen dopant atom on both the magnetic moment and the electric dipole of Pr and $\mathrm{Tb}$ clusters. The oxygen atom can in some cases (smaller $\mathrm{Tb}_{\mathrm{N}}$ clusters) induce a magnetic moment in an otherwise nonmagnetic cluster. Clusters that already possess a magnetic moment are however not significantly affected by an additional oxygen atom. The oxygen atom does however, in many cases, affect the electric dipole of the clusters, causing a large degree of charge localization within the cluster. In Pr the oxygen atom has little to no effect on either the magnetic or electric system, implying a higher degree of stability than the $\mathrm{Tb}$ clusters.

\section{EXPERIMENTAL DETAILS}

Neutral metal clusters are produced in a cryogenically cooled laser vaporization source. A $10 \mathrm{~ns}$ pulse of a doubled neodymium-doped yttrium aluminum garnet laser $(532 \mathrm{~nm})$ is focused onto a target rod of rare earth metal. The laser

\footnotetext{
${ }^{a)}$ Electronic mail: c.vandijk@ science.ru.nl.
}

pulse ablates a plasma of metal vapor which is subsequently quenched by a pulse of cryogenically cooled $(15 \mathrm{~K})$ helium gas. The metal vapor condenses into clusters and the clusters remain in the source long enough to ensure that they are at thermal equilibrium with the buffer gas. After this dwell time, the gas-cluster mixture is expanded through a nozzle into vacuum. The nozzle expansion is skimmed and collimated into a narrow beam. The beam velocity is determined by the temperature of the buffer gas, and is measured using a mechanical chopper.

To measure the magnetic response, the cluster beam is directed in between the pole faces of a Rabi two-wire electromagnet, which applies an inhomogeneous magnetic field of $350 \mathrm{~T} / \mathrm{m}$ in a direction transverse to the beam. To measure the electric response the cluster beam is sent through a coaxial electric deflection plate which applies an inhomogeneous electric field of up to $7 \times 10^{6} \mathrm{~V} / \mathrm{m}$. In both of these cases, the clusters experience a force and are deflected in a direction transverse to the beam. By measuring the deflections we can determine the magnetic moment or electric dipole of the clusters. The clusters are mass separated and the deflections are measured with a position sensitive time-offlight mass spectrometer. ${ }^{1}$

The average magnetization is calibrated using the deflection profile of the $\mathrm{Al}$ atom. The magnetic moment is then calculated from the average magnetization $M$ using the Langevin function, see Eq. (1), which has been shown to be a good approximation for free clusters even though there is no heat bath present for isolated clusters in a vacuum ${ }^{2,3}$

$$
M=\mu\left[\operatorname{coth}\left(\frac{\mu B}{k T}\right)-\frac{k T}{\mu B}\right] \text {. }
$$

In the low field limit (for $\mu B / k T \ll 1$ ), this reduces to

$$
M=\frac{\mu^{2} B}{3 k T} .
$$

By analyzing the electric deflection profiles we can extract two quantities of interest: the shift and the broadening. The shift gives us a measure of the induced electric dipole or 


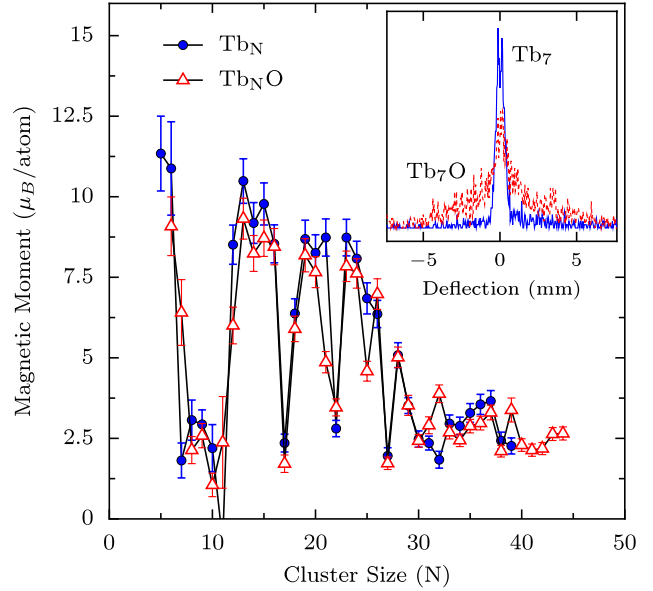

FIG. 1. (Color online) Magnetic moment of $\mathrm{Tb}_{\mathrm{N}}$ clusters with and without an oxygen dopant atom. The addition of an oxygen atom has little effect on the total moment of the cluster; $\mathrm{T}=77 \mathrm{~K}$. Inset: deflection profiles for $\mathrm{Tb}_{7}$ and $\mathrm{Tb}_{7} \mathrm{O}$. The addition of an oxygen atom gives rise to a magnetic moment in an otherwise nonmagnetic cluster.

polarizability of the cluster. The amount of broadening relates to the permanent dipole in the cluster. Here solely the broadening will be treated for further analysis. The total deflection is given in Eq. (3) which includes contributions from both the induced $P=\alpha E$ and permanent $p$ dipole moments ${ }^{4}$

$$
d=\left(\alpha+\kappa \frac{p^{2}}{k T_{R}}\right) \frac{E \nabla E}{m v^{2}} K_{e},
$$

where $T_{R}$ is the rotational temperature of the cluster, $\kappa$ is a constant dependent on the cluster's axes of inertia, and $K_{e}$ is a calibration constant for the deflection field.

\section{RESULTS}

\section{A. Terbium}

The magnetic moment of $\mathrm{Tb}_{\mathrm{N}}$ and oxygen-doped $\mathrm{Tb}_{\mathrm{N}} \mathrm{O}$ clusters are presented in Fig. 1. One can see a striking oscillatory pattern in the moments as a function of cluster size, more than likely due to structural effects such as geometric shells. ${ }^{5}$ The addition of an oxygen dopant atom has little effect on the net moment of the cluster, although it arguably leads to a slight decrease for $\mathrm{N}>10$. In $\mathrm{Tb}_{7}$ however, a cluster with a negligible magnetic moment, the oxygen atom actually causes an increase in the total moment of the cluster. Accompanying this change, the electric dipole increases significantly.

The permanent dipole of $\mathrm{Tb}_{\mathrm{N}}$ clusters is shown in Fig. 2 where a clear increase can be seen with the addition of an oxygen dopant atom. An electric dipole of $\sim 5 \times 10^{-3} \mathrm{D}$ or less can be considered to be negligible and within the measurement limit of the experiment. Thus most of the pure $\mathrm{Tb}$ clusters have no significant permanent electric dipole. The electric dipole then increases significantly with the addition of an oxygen atom to the cluster. The increase of electric dipole is however not consistent across all sizes and peaks for several cluster sizes across the measured size range can be seen. This behavior cannot be easily understood a priori but could possibly be due to structural changes from one cluster size to another. Interestingly, these peaks do not cor-

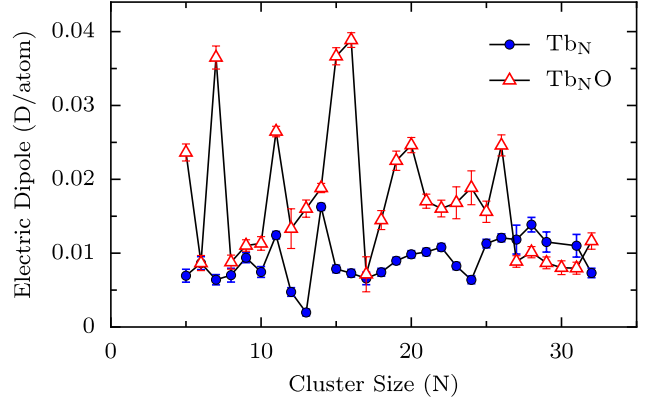

FIG. 2. (Color online) Permanent electric dipole of $\mathrm{Tb}_{\mathrm{N}}$ clusters with and without an oxygen dopant atom. Note the increase in dipole moment with the addition of an oxygen atom; $\mathrm{T}=23 \mathrm{~K}$.

respond to any features in the magnetic moments seen in Fig. 1.

\section{B. Praseodymium}

The magnetic moment of Pr clusters, as seen in Fig. 3, behaves quite differently than that of the Tb clusters. An odd-even oscillation is present for many of the cluster sizes, indicative of an antiferromagnetic ordering. There is also a large peak at $\operatorname{Pr}_{13}$, something which can be attributed to a HCP-structured cluster with high symmetry and coordination. ${ }^{6}$ As with $\mathrm{Tb}$, the magnetic moments of $\operatorname{Pr}_{\mathrm{N}} \mathrm{O}$ clusters do not differ significantly to the undoped clusters.

Also of note here is that $\mathrm{Pr}$ is in the bulk nonmagnetic, due to its large crystal field splitting. ${ }^{7}$ Experiments by McEwen $e t a l .{ }^{8}$ showed that by inducing strain on single crystal of Pr, spontaneous magnetic ordering can occur. Similarly, in clusters the arrangement of the atoms is different than in the bulk, and thus the crystal field within the cluster also differs. Therefore magnetic ordering is here possible in an otherwise nonmagnetic material.

The permanent dipole of $\operatorname{Pr}_{\mathrm{N}}$ clusters is given in Fig. 4, and here no noticeable difference can be observed with the addition of an oxygen dopant atom. This is in contrast to $\mathrm{Tb}$ where a significant increase is seen in the oxygen-doped clusters. Here both $\operatorname{Pr}_{\mathrm{N}}$ and $\operatorname{Pr}_{\mathrm{N}} \mathrm{O}$ can largely be considered as having no permanent electric dipole, excluding a few particular clusters such as $\operatorname{Pr}_{10}$ and $\operatorname{Pr}_{8} \mathrm{O}$.

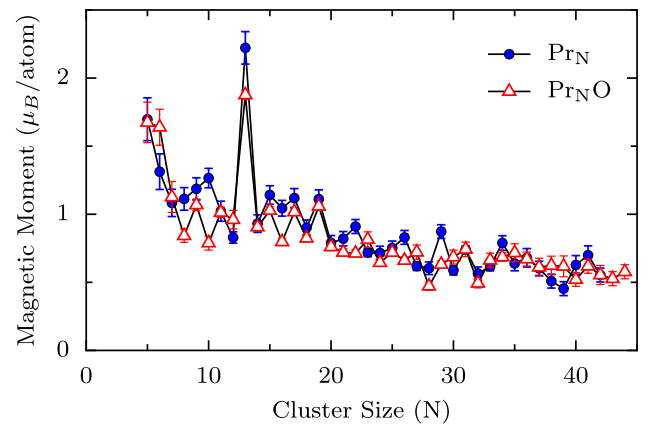

FIG. 3. (Color online) Magnetic moment of $\operatorname{Pr}_{\mathrm{N}}$ clusters with and without an oxygen dopant atom. The addition of an oxygen atom has little effect on the total moment of the cluster; $\mathrm{T}=50 \mathrm{~K}$. 


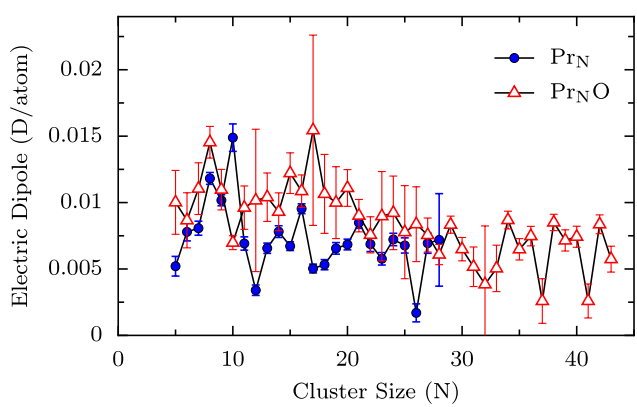

FIG. 4. (Color online) Permanent electric dipole of $\operatorname{Pr}_{N}$ clusters with and without an oxygen dopant atom. The addition of an oxygen atom has little effect on the total dipole of the cluster; $\mathrm{T}=26 \mathrm{~K}$.

\section{DISCUSSION AND CONCLUSION}

As we have shown here for the case of $\mathrm{Tb}_{\mathrm{N}} \mathrm{O}$ clusters, the addition of an oxygen dopant atom leads to a significantly increased permanent electric dipole. This is in contrast to $\operatorname{Pr}_{\mathrm{N}} \mathrm{O}$ where the effect is less significant. Correspondingly there is little change in the magnetic moment of $\operatorname{Pr}_{N}$ with the addition of an oxygen atom. The additional oxygen atom can affect the net magnetization of the cluster in two possible ways: it can (1) cause a change in the charge distribution within the cluster or (2) induce a structural change reorienting the relative positions of the atoms within the cluster. While it is likely that some structural changes do take place, as seen by some anomalous sizes in both $\mathrm{Tb}$ and $\mathrm{Pr}$, by looking at the electric dipole data of $\mathrm{Tb}_{\mathrm{N}} \mathrm{O}$ we can directly see that the charge distribution within the cluster has also been affected. This however happens sporadically on a per cluster basis while the changes in the magnetic moment (if any) do not follow a similar pattern.
It is known that the exchange interaction in rare earth metals in the bulk between neighboring localized $4 f$ spins is mediated by the delocalized $5 d$ electrons, as described by the RKKY exchange mechanism. ${ }^{7}$ What we see here however is that there is little correlation between an increase in the electric dipole of a cluster and its total magnetic moment. Note that the presence of a permanent electric dipole excludes the possibility of any free "metal-like" electrons in the cluster. This raises the question as to the mechanism of exchange in these small systems as differences in charge density do not seem to significantly affect the exchange interaction, such as in $\mathrm{Tb}_{\mathrm{N}} \mathrm{O}$. This brings into question the validity of the RKKY exchange mechanism in small systems.

\section{ACKNOWLEDGMENTS}

Funding for this research was provided by the National Science Foundation (NSF), the Dutch Nanotechnology initiative NanoNed and EC FP7 contributions under Grant Nos. NMP3-SL-2008-214469 (UltraMagnetron) and N 214810 (FANTOMAS).

${ }^{1}$ W. A. de Heer and P. Milani, Rev. Sci. Instrum. 62, 670 (1991).

${ }^{2}$ X. Xu, S. Yin, R. Moro, and W. A. de Heer, Phys. Rev. Lett. 95, 237209 (2005).

${ }^{3}$ X. Xu, S. Yin, R. Moro, and W. A. de Heer, Phys. Rev. B 78, 054430 (2008).

${ }^{4}$ W. A. de Heer and V. V. Kresin, e-print arXiv:0901.4810.

${ }^{5}$ H. Haberland, T. Hippler, J. Donges, O. Kostko, M. Schmidt, and B. von Issendorff, Phys. Rev. Lett. 94, 035701 (2005).

${ }^{6}$ D. P. Pappas, A. P. Popov, A. N. Anisimov, B. V. Reddy, and S. N. Khanna, Phys. Rev. Lett. 76, 4332 (1996).

J. Jensen and A. Mackintosh, Rare Earth Magnetism (Clarendon, Oxford, 1991).

${ }^{8}$ K. A. McEwen, W. G. Stirling, and C. Vettier, Phys. Rev. Lett. 41, 343 (1978). 